Check for updates

Cite this: Chem. Sci., 2020, 11, 224

๑ All publication charges for this article have been paid for by the Royal Society of Chemistry

\title{
Improving the reactivity of hydrazine-bearing MRI probes for in vivo imaging of lung fibrogenesis $\uparrow$
}

\author{
Eman A. Akam, (iD abc Eric Abston, ade Nicholas J. Rotile, ${ }^{\text {ab }}$ Hannah R. Slattery, ${ }^{\text {ab }}$ \\ Iris Y. Zhou, ${ }^{\text {abc }}$ Michael Lanutice and Peter Caravan (D)*abc
}

Pulmonary fibrosis (PF) is the pathologic accumulation of extracellular matrix components in lung tissue that result in scarring following chronic lung injury. PF is typically diagnosed by high resolution computed tomography (HRCT) and/or invasive biopsy. However, HRCT cannot distinguish old injury from active fibrogenesis. We previously demonstrated that allysine residues on oxidized collagen represent an abundant target during lung fibrogenesis, and that magnetic resonance imaging (MRI) with a small-molecule, gadolinium-containing probe, Gd-Hyd, could specifically detect and stage fibrogenesis in a mouse model. In this work, we present an improved probe, Gd-CHyd, featuring an N,Ndialkyl hydrazine which has an order of magnitude both greater reactivity and affinity for aldehydes. In a paired study in mice with bleomycin induced lung injury we show that the improved reactivity and affinity of Gd-CHyd results in significantly higher lung-to-liver contrast, e.g. 77\% higher at 45 min post injection, and slower lung clearance than Gd-Hyd. Gd-CHyd enhanced MRI is >60-fold higher in bleomycin injured mouse lungs compared to uninjured mice. Collectively, our data indicate that enhancing hydrazine reactivity and affinity towards allysine is an effective strategy to significantly improve molecular MRI probes for lung fibrogenesis.

Received 24th September 2019
Accepted 8th November 2019

DOI: $10.1039 / c 9 s c 04821 a$

rsc.li/chemical-science and pattern of lung scarring but cannot determine current disease activity or predict disease progression. ${ }^{7}$ Progression of IPF to its end stage can take several clinical forms from rapid or slow decline to stable disease punctuated with periods of rapid decline. ${ }^{8}$ Assessment of early disease stages of IPF with HRCT is further complicated because radiological patterns that determine disease are typically those occurring at late stages of the disease. ${ }^{9}$ The limitations of HRCT in IPF diagnosis along with the unpredictability of disease progression severely limit patient prognostication. A noninvasive method to identify patients with disease, to identify regions of active disease activity, and/or to identify patients likely to progress rapidly would also have benefit in developing new therapeutics for IPF because patients could be better stratified for trial enrolment, and early measures of treatment response may be observed. ${ }^{\mathbf{1 0 - 1 3}}$

We are interested in developing molecular magnetic resonance (MR) probes to asses IPF disease activity by targeting biochemical features of active fibrosis (i.e. fibrogenesis). MR imaging is a non-invasive technique that provides threedimensional, high resolution anatomical images without ionizing radiation. Coupled with a targeted MR visible probe, molecular imaging can be used to specify disease location and activity, quantify extent of disease and monitor response to therapy. Molecular MR probes can detect unique biochemical and cellular markers of disease and allow for early assessment and diagnosis. ${ }^{\mathbf{1 4 , 1 5}}$ Fibrosis is accompanied by upregulation of lysyl oxidase (Lox) and Lox-like (LoxL) enzymes ${ }^{\mathbf{1 6}}$ which catalyze 
oxidation of lysine $\varepsilon$-amino groups to allysine aldehydes thereby allowing for the crosslinking of collagen and formation of scar. ${ }^{17}$ The electrophilic aldehydes of allysine residues provide an abundant target for reaction with nucleophiles and thus a mechanism by which MRI probes can be targeted to fibrosing tissue. ${ }^{18,19}$

Hydrazines selectively react with aldehydes and ketones to form hydrazones under physiological conditions with water being the sole by-product. Hydrazines present excellent reactive handles for bioorthogonal reactions linking targets and probes to biomolecules of interest. ${ }^{20}$ Recently a turn-on chemical exchange saturation transfer (CEST) MR probe that detects small bioactive aldehydes in vitro was reported, ${ }^{21}$ as well as some aldehyde targeting positron emission tomography probes. ${ }^{22,23}$ We have demonstrated that the hydrazine-equipped aldehyde-reactive small molecule gadolinium chelate Gd-Hyd can bind aldehydes in vivo and robustly stage and quantify hepatic and pulmonary fibrogenesis. ${ }^{18}$

Gd-Hyd is hydrophilic, anionic, and exhibits very low nonspecific protein binding which results in a short, 5 minute blood half-life in vivo in mice. ${ }^{18}$ This short residency time results in efficient whole body elimination and low background signal, however it does limit the time that the complex is exposed to target tissue. Increasing the rate of reaction with aldehydes should lead to an increase of the on-target accumulation of the probe. Extensive work by Kool and co-workers on the reactivity of hydrazines reveals that electron-poor acyl hydrazines such as that featured in Gd-Hyd react with aldehydes at a slower rate than electron-rich alkyl hydrazines. ${ }^{\mathbf{2 0 , 2 4 , 2 5}}$ As such, we sought to improve the reactivity of the prototype probe Gd-Hyd by substituting the reactive hydrazine moiety from a hydrazide to alkyl hydrazine. In this work, we present the aldehyde-reactive gadolinium chelate Gd-CHyd, featuring a secondary alkyl hydrazine. We compare the in vitro reactivity of Gd-CHyd to that of Gd-Hyd and assess the consequences of the differing reactivities in vivo in a bleomycin (BM) lung-injury model of lung fibrosis.

\section{Results and discussion}

\section{Synthesis and reactivity of Gd-CHyd with aldehydes}

The allysine-reactive probe Gd-CHyd features a Gd-DOTA scaffold conjugated to a hydrazine arm that reacts reversibly with aldehydes (Fig. 1a). Unlike the prototype probe Gd-Hyd (Fig. 1a), ${ }^{18}$ Gd-CHyd features an electron-rich piperazinohydrazine which we hypothesized would undergo condensation reactions at a faster rate than the acyl hydrazide of Gd-Hyd (Fig. 1a). As depicted in Scheme 1, we first synthesized the carboxybenzyl (CBZ)-protected piperazino-hydrazine arm 1 via an alkylation/cyclization reaction of bis(2-chloroethyl)amine hydrochloride with benzyl carbazate. We then prepared the activated ester of $t$-butyl protected DOTAGA using $N$-hydroxysuccinamide (NHS) and $N, N^{\prime}$-dicyclohexylcarbodiimide (DCC) and coupled the active ester with $\mathbf{1}$ to give compound 2 . Removal of the $t$-butyl groups using trifluoroacetic acid resulted in the CBZ-protected scaffold 3 which we used to chelate gadolinium resulting in $\mathbf{4}$. Finally, a hydrogenolysis reaction
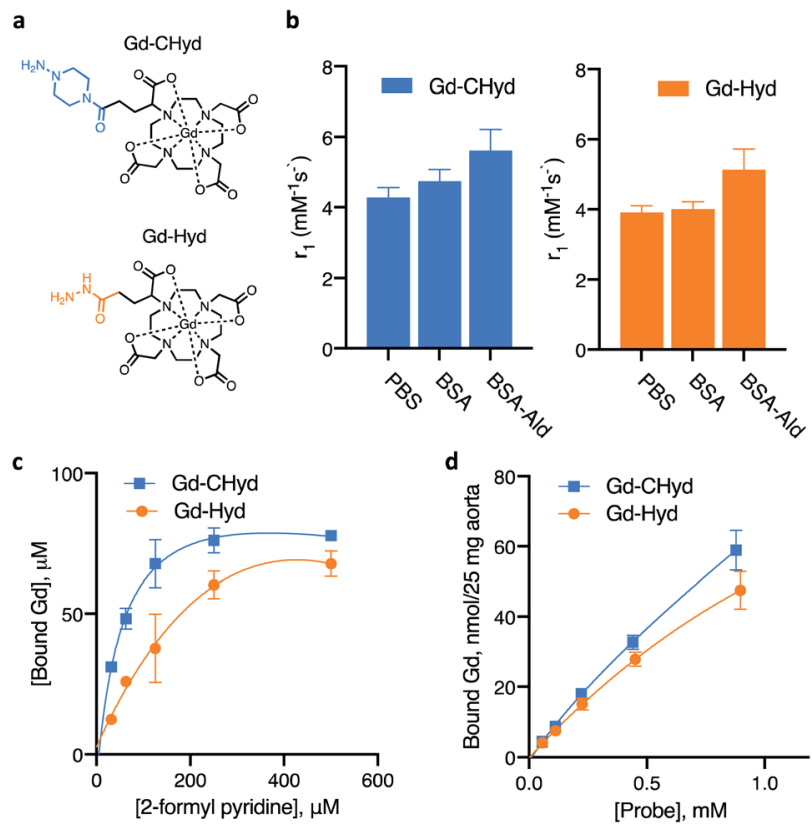

Fig. 1 Conjugation of the probes to 2-formyl pyridine, allysine-rich protein (BSA-Ald) and allysine-rich tissue (porcine aorta). (a) Structures of probes evaluated. (b) Relaxivity values at $60 \mathrm{MHz}$ (PBS, pH 7.40, 37 ${ }^{\circ} \mathrm{C}$ ) for $\mathrm{Gd}-\mathrm{Hyd}$ and $\mathrm{Gd}-\mathrm{CHyd}$ in the presence or absence of BSA and aldehyde-rich BSA-Ald. (c) Concentration-dependent binding of the probes to 2-formyl pyridine as determined by LC-ICP-MS (d) binding of probes to allysine-rich porcine aorta as determined by ICP-MS following tissue digestion.

using catalytic amounts of palladium on carbon resulted in the final product Gd-CHyd (5).

We compared the rates of reactivity of Gd-CHyd and Gd-Hyd with a small-molecule model aldehyde under physiologically relevant conditions. We chose 2 -formyl pyridine in order to

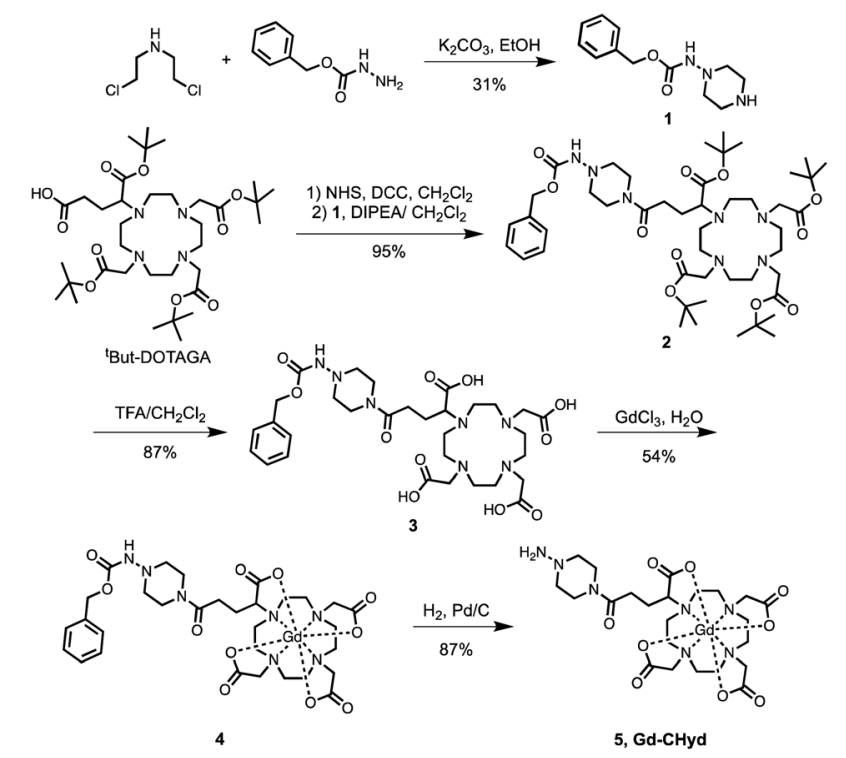

Scheme 1 Synthetic route of the aldehyde-reactive Gd-CHyd. Charges on Gd-complexes are omitted for clarity. 
follow the condensation reaction spectrophotometrically as previously reported. ${ }^{24}$ We found that Gd-CHyd reacts with 2formyl pyridine an order of magnitude faster than Gd-Hyd (Table 1) under pseudo-first order conditions at physiological $\mathrm{pH}$ and temperature. We confirmed the relative rates of reactivity of the two probes using a chromatographic method with ICP-MS detection under slightly different conditions and found that the relative reactions rates remain consistent (ESI, Fig S5 and Table S1 $\dagger$ ).

The extent of formation of the condensation product, i.e. the equilibrium dissociation constant $K_{\mathrm{d}}$, is another factor that should impact the accumulation of the probe in fibrosing tissue. We evaluated the dissociation constant for the reaction of Gd-Hyd and Gd-CHyd with 2-formyl pyridine at various concentrations. We quantified the amount of Gd-containing species using a chromatographic method with ICP-MS detection and calculated the corresponding dissociation constants (Fig. 1c). We found that the dissociation constants for these reactions fall within literature-reported ranges, ${ }^{26}$ with $K_{\mathrm{d}}$ for GdCHyd being 9-fold smaller than that for Gd-Hyd (Table 1).

We then examined the reaction of Gd-Hyd with an aldehydebearing protein, oxidized bovine serum albumin (BSA-Ald) and evaluated the effect of protein-conjugation on relaxivity (ability to effect MR signal change) in solution. BSA is a soluble, lysine rich protein that can serve as a useful model. We oxidized BSA using reported procedures and quantified the aldehyde load, confirming the presence of one aldehyde motif per protein $(\mathrm{ESI} \dagger)^{27,28}$ We then determined the relaxivity of Gd-Hyd or GdCHyd after $72 \mathrm{~h}$ incubation with BSA, BSA-Ald or in the absence of protein. In PBS at $1.41 \mathrm{~T}$ and $37^{\circ} \mathrm{C}$, Gd-Hyd and GdCHyd exhibit similar relaxivities of $3.9 \pm 0.2$ and $4.3 \pm 0.3$ $\mathrm{mM}^{-1} \mathrm{~s}^{-1}$ respectively, decreasing slightly at $4.7 \mathrm{~T}$ to $3.4 \pm 0.1$ and $4.1 \pm 0.1 \mathrm{mM}^{-1} \mathrm{~s}^{-1}$ respectively. At $1.41 \mathrm{~T}$ in the presence of unmodified BSA protein, the relaxivity increases slightly due to non-specific protein interaction. Incubation of Gd-Hyd or GdCHyd with BSA-Ald resulted in a $30 \%$ increase of relaxivity due to covalent interaction with protein aldehydes (Fig. 1b). We confirmed binding of probes to BSA-Ald by passing the sample though an ultracentrifugation 5000 Da filter and quantifying the unbound gadolinium probe in the filtrate by ICP-MS (ESI, Fig. S7†).

The aorta is a tissue rich in allysine because it is constantly being remodelled due to high shear stress. ${ }^{28,29}$ We evaluated the binding of Gd-Hyd and Gd-CHyd to porcine aorta to assess the

Table 1 Kinetic and thermodynamic parameters measured for the condensation reactions of hydrazine-bearing gadolinium probes. The pseudo-first order rate constant $\left(k_{\text {obs }}\right)$ for the reaction $\mathrm{Gd}-\mathrm{CHyd}$ with 2-formyl pyridine at $\mathrm{pH} 7.40$ in PBS is 11 times larger than that measured for $\mathrm{Gd}$-Hyd. The $\mathrm{K}_{\mathrm{d}}$ for this reaction indicates that the $\mathrm{Gd}$ CHyd hydrazone is 9 times more stable than the analogous $\mathrm{Gd}-\mathrm{Hyd}$ hydrazone

\begin{tabular}{lllrl}
\hline & $k_{\text {obs }},\left(\mathrm{min}^{-1}\right)$ & Relative $k_{\text {obs }}$ & $K_{\mathrm{d}}(\mu \mathrm{M})$ & Relative $K_{\mathrm{d}}$ \\
\hline Gd-hyd & $0.0028 \pm 0.0006$ & 1 & $415 \pm 63$ & 1 \\
Gd-CHyd & $0.0314 \pm 0.0002$ & 11 & $45 \pm 16$ & 0.11
\end{tabular}

concentration-dependent conjugation of probe in allysine-rich tissue at equilibrium. We found that the amount of probe bound to porcine aorta after $72 \mathrm{~h}$ incubation was higher for GdCHyd than for Gd-Hyd but neither binding isotherm reached saturation (Fig. 1d).

Collectively, these data demonstrate that Gd-CHyd reacts with small molecule aldehydes in solution, aldehyde-containing proteins in solution, and aldehyde-rich tissue. Gd-CHyd undergoes hydrazone condensation reactions at an order of magnitude faster rate than Gd-Hyd, and Gd-CHyd results in an order of magnitude more thermodynamically stable hydrazone product than Gd-Hyd.

\section{Bleomycin lung injury mouse model of lung fibrogenesis}

We evaluated the effects of the superior reactivity of Gd-CHyd in vivo in the bleomycin-induced lung-injury model (BM) in mice. We previously demonstrated that Gd-Hyd can quantify and stage pulmonary fibrogenesis in the lungs of BM mice, and that Gd-Hyd enhanced MRI can be used to monitor disease progression and response to treatment of $\mathrm{PF} .{ }^{18}$ We sought to directly compare Gd-CHyd enhanced lung MRI to that generated by Gd-Hyd. The BM model is known for its heterogeneity both in location of fibrosis and extent of disease. We therefore designed a pair-wise study to mitigate the heterogeneity of the disease in this model. We imaged mice 14 days after initial bleomycin injury first with Gd-Hyd and then $24 \mathrm{~h}$ later we performed the same imaging protocol in the same mouse with GdCHyd (Fig. 2a). After the second imaging session, the mice were euthanized and the lungs and other tissues harvested for analysis. We chose to image with Gd-CHyd second in the twoprobe sequence in order to determine the biodistributions of this new probe while adhering to the $3 \mathrm{R}$ principles in the ethical

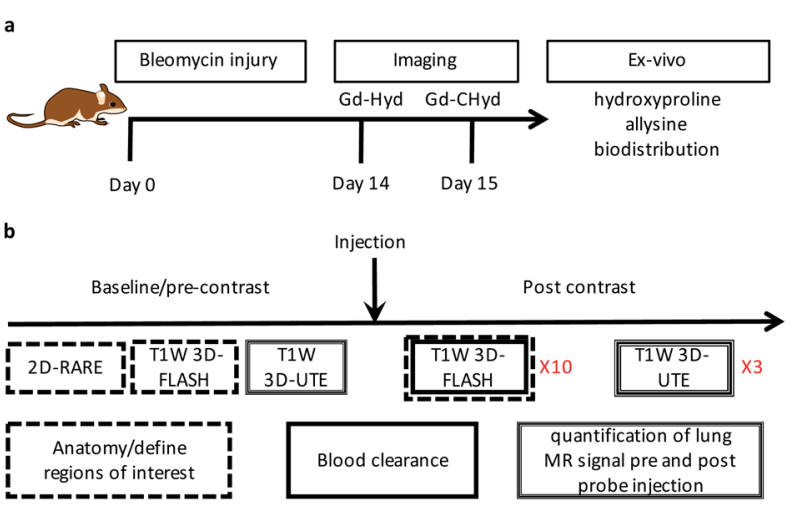

Fig. 2 Study time line and imaging sequences (a) Bleomycin administration to initiate lung injury at day 0 followed by 14 days of fibrosis development. On day 14, the animals are imaged dynamically with GdHyd. After 24 hours, the same animals are dynamically imaged with $\mathrm{Gd}-\mathrm{CHyd}$, and then euthanized and the organs collected for analyses. (b) Anatomical 2D-RARE and T1-weighted (T1W) 3D-FLASH images are acquired before probe injection and are used along with the 3D-FLASH images post injection to define regions of interest (ROIs). The T1W 3DUTE images are used to quantify the signal in the lung ROIs before and after probe injection. The same imaging protocol was followed for a cohort with bleomycin-naive mice that were imaged with $\mathrm{Gd}-\mathrm{CHyd}$ only. 
use of animals: replacement, reduction, and refinement. ${ }^{30}$ We verified complete clearance of Gd-Hyd from bleomycin-injured lungs 24 hours post injection by harvesting the tissue and determining the gadolinium content by ICP-MS. We found that the lung gadolinium concentration 24 hours post Gd-Hyd injection was below the detection limit of our instrument. We also imaged BM mice $(n=2)$ with Gd-Hyd on two consecutive days and found that there was no significant change in the MR signal obtained on the different days. This confirms that disease progression within 24 hours is negligible.

We verified fibrosis in BM animals by histology and by quantifying the biochemical markers hydroxyproline and allysine in the lungs of naïve and BM mice. After imaging, the right lung was fixed in formalin and stained with hematoxylin and eosin (H\&E) and sirius red/fast green (S/F) (Fig. 3a). H\&E staining show increased tissue density and cellularity in the bleomycin injured lungs. S/F staining shows increased regions of tissue stained red, indicative of fibrosis. We quantified the area of the slides that stained positive with $\mathrm{S} / \mathrm{F}$ as the collagen proportional area (CPA, Fig. 3b), and observed a 10-fold and significantly higher CPA in the bleomycin injured lungs. The left lungs of the animals were homogenized and the amounts of hydroxyproline and allysine were determined using previously reported assays. ${ }^{\mathbf{1 8 2 8}}$ Hydroxyproline is a quantitative marker of total collagen in fibrosing tissue commonly used to gauge the severity of fibrosis. Allysine is the aldehyde target for Gd-Hyd and Gd-CHyd and is formed from the oxidation of collagen lysine through lysyl oxidase enzymes. ${ }^{29} \mathrm{We}$ found that the lungs of BM mice had significantly higher levels of collagen, hydroxyproline and allysine compared to the lungs of naïve mice
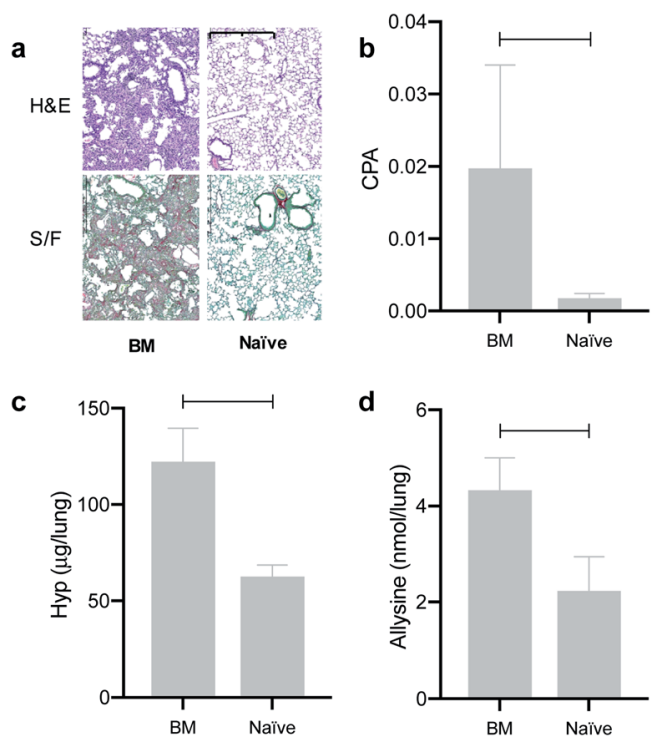

Fig. 3 Ex vivo assessment of lung fibrosis. (a) Hematoxylin and eosin (H\&E)- and sirius red/fast green (S/F)-stained sections of lung tissues from mice that were challenged with bleomycin intratracheally 2 weeks prior (BM, $n=8$ ) or naïve mice $(n=4)$ ). (b) Lung injury as assessed by collagen proportional area (CPA), (c) lung hydroxyproline content and (d) allysine content. $* p<0.05, * * p<0.01, * * * p<0.001, t-$ test. Scale bar $=1 \mathrm{~mm}$.
(Fig. 3). Confirming the presence of fibrosing tissue in bleomycin-injured lungs.

In the imaging protocol, we first acquired 2D Rapid Acquisition with Relaxation Enhancement (RARE) images, T1weighted 3D Fast Low Angle Shot (FLASH) images, and 3D T1weighted ultrashort time to echo (UTE) images. Mice were then injected with probe $\left(0.1 \mathrm{mmol} \mathrm{kg} \mathrm{kg}^{-1}\right)$ as a bolus via an indwelling tail vein line. Then the FLASH sequence was repeated 10 times for a total of 15 minutes to follow clearance of the probe and to measure the blood half-life. The UTE sequence was repeated at 15,30 , and 45 minutes after probe injection. After the last UTE acquisition, the mouse was removed from the scanner. The UTE sequence overcomes the inherently low MR signal in the lung due to the very short $\mathrm{T} 2 *$ of the lung protons. However, the UTE images do not provide strong contrast between the lung parenchyma, large vessels in the lung, or airways. Therefore, we used the RARE (black blood, black lung) and the first post probe FLASH (bright blood) images to define regions of interest (ROIs) in the lung that excluded vessels and airways. A total of 8 lung ROIs were defined on sagittal image slices spanning both lungs. As a reference tissue, we also defined ROIs in the liver in each slice. We averaged the lung (or liver) signal from the 8 slices to get signal intensity (SI) at a given time point. We computed the lung-to-liver contrast-to-noise ratio as $\left(\mathrm{SI}_{\text {lung }}-\mathrm{SI}_{\text {liver }}\right) / \mathrm{SD}_{\text {air }}$, where $\mathrm{SD}_{\text {air }}$ is the standard deviation in an ROI drawn outside of the animal. We then calculated the change in CNR $(\Delta \mathrm{CNR})$ at each time point post probe injection compared to the image before probe injection.

We found that the signal generated by Gd-CHyd was significantly higher than that generated by Gd-Hyd at all three time points post injection of contrast agent (Fig. 4a). Notably, the percent difference in $\Delta \mathrm{CNR}$ between Gd-Hyd and Gd-CHyd increases with time, i.e. $34 \%$ after 15 minutes, 53\% after 30 minutes and $77 \%$ after 45 minutes indicating slower washout from the fibrotic lung with Gd-CHyd compared to Gd-Hyd. Since the same animal was imaged with both probes, we could also compare the pairwise effect (Fig. 4b). We found that the lung
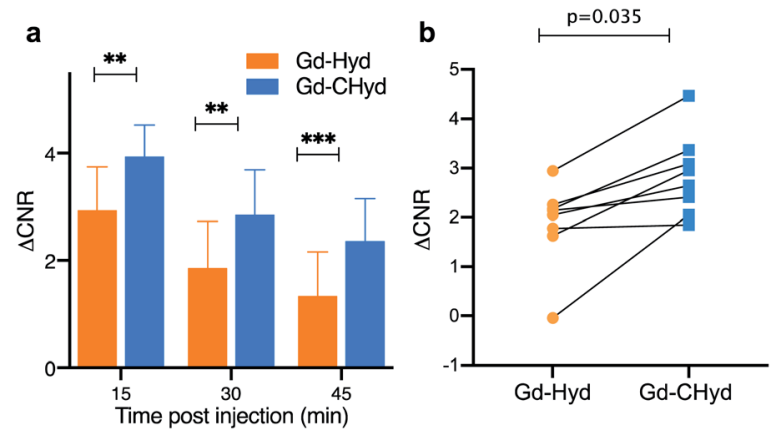

Fig. 4 Quantification of the change in lung-to-liver CNR of BM mice after injection of Gd-Hyd or Gd-CHyd 14 and 15 days after bleomycin injury, respectively. Mice were imaged prior to and 15, 30 and $45 \mathrm{~min}$ post probe injection. (a) Gd-CHyd lung enhancement is always greater than Gd-Hyd enhancement at all three time points post contrast ( $n=$ 8). (b) Pair-wise analysis of the $\triangle C N R$ in the lungs of $B M$ animals observed arising from Gd-Hyd or Gd-CHyd injection. $* * p<0.05, * * * p$ $<0.001$, paired $t$-test. 
signal from Gd-CHyd enhanced MRI was higher than that from Gd-Hyd enhanced MRI for all eight animals.

There was very little signal enhancement in other tissues. Gd-CHyd undergoes rapid clearance from the blood through the kidneys with a half-life of $5.6 \pm 1.3 \mathrm{~min}$, which was not statistically significantly different than the $4.7 \pm 1.7 \mathrm{~min}$ blood halflife measured for Gd-Hyd. ${ }^{18}$ Apart from the initial transient increase in signal immediately post injection due to blood distribution, there was no increase in signal in the liver consistent with exclusive renal elimination (ESI, Fig. S9†). Because Gd-Hyd and Gd-CHyd have similar structures, blood half-lives, in vivo clearance routes (renal), and are both stable in blood plasma (ESI, Fig S6†), the lung imaging results collectively indicate that Gd-CHyd has greater uptake and persists longer in fibrosing lung tissue compared to Gd-Hyd. We also compared the lung MR signal and $\triangle \mathrm{CNR}$ using Gd-CHyd enhanced MRI of naïve (control) mice. We found that there was a remarkable difference in the signal in the lungs of BM animals compared to the lungs of naïve animals 30 minutes after injection of Gd-CHyd (Fig. 5a)

In naive mice, there is essentially no lung enhancement with Gd-CHyd as expected. MR images unambiguously reflect the notable contrast enhancement in the lungs of BM animals injected with Gd-CHyd compared to the same animal injected with Gd-Hyd (Fig. 5c) and the same differences are visually observable in the case of BM vs. naïve animals imaged with GdCHyd. Notably, we observed a 12 -fold enhancement in $\Delta \mathrm{CNR}$ in the lungs of BM animals compared to naïve animals $15 \mathrm{~min}$
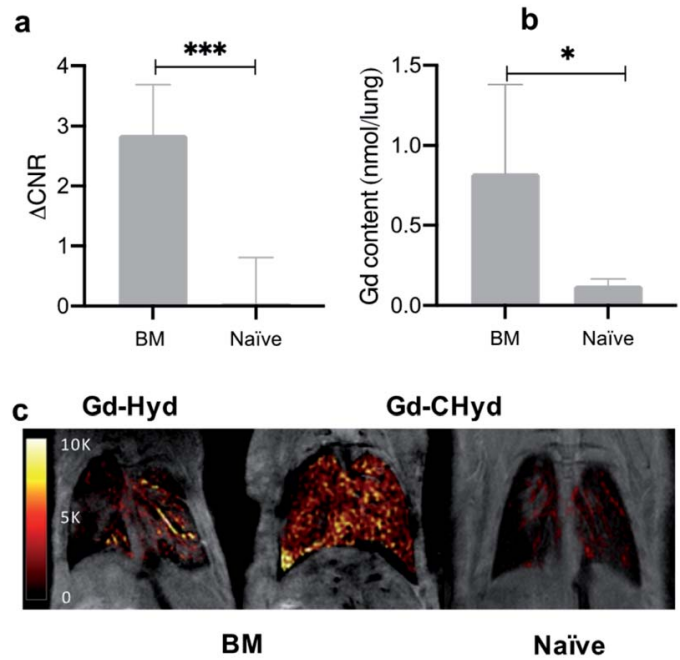

Fig. 5 Selectivity of Gd-CHyd for actively fibrosing lung vs. healthy lung. (a) Quantification of the MR signal in the lungs of bleomycininjured (BM, $n=8$ ) mice or naïve mice (naïve, $n=4) 30$ min after GdCHyd administration revealing 63-fold higher signal in bleomycininjured lungs. (b) Quantification of the gadolinium content in the left lungs of BM or naive animals 75 minutes post injection of $\mathrm{Gd}-\mathrm{CHyd}$ showing 10-fold higher amounts of gadolinium in the lungs of BM mice. (c) Coronal RARE MR images overlaid with false color image of lung enhancement generated by subtraction of the pre-injection UTE image from the post injection UTE image. Enhancement is highest in the lungs of BM-treated mouse injected with Gd-CHyd. $* p<0.05$, $* * * p<0.001$, unpaired $t$-test. post injection of Gd-CHyd (ESI, Fig S10 $\dagger$ ). In a previous report, Gd-Hyd showed only a 3.7 -fold enhancement in $\Delta$ CNR in the lungs of BM animals compared to naïve animals at a similar time point (12 $\mathrm{min}$ post injection). ${ }^{18}$

We quantified the gadolinium content in the left lungs of mice 75 min post Gd-CHyd administration. We found that the lung gadolinium content of $\mathrm{BM}$ mice was 10 times higher than that measured in naïve animals, in line with the observed MR signal changes (Fig. 5b)

\section{Conclusions}

Gd-CHyd is an improved allysine-targeted small molecule MRI molecular probe suitable for imaging lung fibrogenesis. Gd-CHyd is equipped with a secondary alkyl hydrazine which resulted in an 11-fold increase in aldehyde binding rate constant and an order of magnitude higher affinity for aldehydes compared to our earlier prototype the acyl-hydrazine Gd-Hyd which employed an acyl hydrazide for targeting. The relaxivities of Gd-Hyd and GdCHyd were similar and increased by $10 \%$ when conjugated to the aldehyde groups of proteins. Both Gd-CHyd and Gd-Hyd 60fold higher signal in bleomycin-injured lungs, exhibit rapid blood clearance through the kidneys with very low signal enhancement in the liver, heart, muscle, or normal lung tissue. The greater aldehyde affinity and reactivity of Gd-CHyd translates in significantly higher lung-to-liver $\Delta \mathrm{CNR}$ values than Gd-Hyd at $15 \mathrm{~min}$ post injection (30\% higher) and at $45 \mathrm{~min}$ post injection $(77 \%$ higher). We previously reported MR probes and methods to quantify the burden of fibrosis in the lung, ${ }^{31}$ inflammation in the lung, ${ }^{32}$ as well as vascular permeability in the context of IPF., ${ }^{33,34}$ Our data indicate that improving the aldehyde reactivity and affinity of hydrazine-bearing gadolinium contrast agents translates to enhanced and prolonged accumulation in fibrosing lung tissue and improved specificity. We are currently expanding our library of allysine-reactive probes to determine the relationship of modifying the rate of reactivity on in vivo efficacy.

\section{Experimental methods}

\section{General methods and instrumentation}

Detailed descriptions, purification methods, characterizations, assay procedures, and imaging sequences can be found in the ESI. $\dagger$

NMR spectra were recorded on a JEOL ECZ 500R 11.7 T NMR system equipped with a $5 \mathrm{~mm}$ broadband probe $\left({ }^{1} \mathrm{H}: 499.81\right.$ $\left.\mathrm{MHz},{ }^{13} \mathrm{C}: 125.68 \mathrm{MHz}\right)$. HPLC-MS purity analysis was carried out on an Agilent 1260 system coupled to Agilent 6130 LC-MS system. Quantification of gadolinium was carried out using an Agilent 8800-QQQ ICP-MS system. HPLC-ICP-MS was carried out on an Agilent 1260 HPLC system coupled to an Agilent 8800QQQ ICP-MS system. Longitudinal (T1) relaxation measurements were recorded using a Bruker mq60 Minispec at $1.41 \mathrm{~T}$ and $37{ }^{\circ} \mathrm{C}$. UV-Vis spectra were recorded on SpectraMax® plus instrument. High resolution mass spectra were acquired with a high-resolution time-of-flight mass spectrometer (AccuTOFDART, JEOL). Mice were imaged on a 4.7 Tesla MRI scanner (Bruker, Billerica MA) using a custom-built volume coil. 
Gd-Hyd and 2-(R)-2-(4,7,10-tris-tert-butylcarboxymethyl1,4,7,10-tetraazacyclododec-1-yl)-pentanedioic acid-1tertbutylester ( ${ }^{t}$ butyl-DOTAGA) were synthesized as previously described. ${ }^{18,35}$ All other reagents were obtained from commercial sources and used without additional purification.

\section{Synthetic procedures}

Synthesis of 1. Bis(2-chloroethyl)amine hydrochloride $(1 \mathrm{~g}$, $5.6 \mathrm{mmol}$ ) was combined with benzyl carbazate $(1.0 \mathrm{~g}, 5.9$ $\mathrm{mmol})$ and potassium carbonate $(2.4 \mathrm{~g}, 16.8 \mathrm{mmol})$ and suspended in ethanol $(20 \mathrm{~mL})$. The suspension brought to reflux under argon for $18 \mathrm{~h}$. Following removal of the resulting precipitate by filtration, the reaction mixture was concentrated under reduced pressure and then purified via Combiflash method 4 with $\mathrm{C}$ and $\mathrm{D}(\mathrm{ESI} \dagger)$ as solvents to give 1 as an offwhite solid (408 mg, 31\% yield). ${ }^{1} \mathrm{H}$ NMR $\left(\mathrm{D}_{2} \mathrm{O}\right): \delta 7.28(\mathrm{~m}$, $5 \mathrm{H}), 5.14(\mathrm{~s}, 2 \mathrm{H}), 3.36(\mathrm{t}, J=5.0 \mathrm{~Hz}, 4 \mathrm{H}), 3.06(\mathrm{t}, J=5.0 \mathrm{~Hz}, 4 \mathrm{H})$. ${ }^{13} \mathrm{C}$ NMR $\left(126 \mathrm{MHz}, \mathrm{D}_{2} \mathrm{O}\right) \delta 160.07,138.9,131.75,131.47130 .74$, 70.21, 54.52, 46.07. LC-MS (method 1, ESI $\dagger$ ): $t_{\mathrm{R}}=2.68 \mathrm{~min}$, HRMS $m / z:[\mathrm{M}+\mathrm{H}]^{+}$calcd for $\left[\mathrm{C}_{12} \mathrm{H}_{18} \mathrm{~N}_{3} \mathrm{O}_{2}\right]^{+}, 236.1399$; found 236.1397.

Synthesis of 2. DOTAGA (100 mg, $0.14 \mathrm{mmol}$ ) was combined with $N$-hydroxysuccinimide (NHS, $18.1 \mathrm{mg}, 0.15 \mathrm{mmol}$ ) and $N, N^{\prime}$-dicyclohexylcarbodiimide (DCC, $29.4 \mathrm{mg}, 0.14 \mathrm{mmol}$ ) and purged with argon. Anhydrous dichloromethane $(2 \mathrm{~mL})$ was added and reaction mixture was stirred for $10 \mathrm{~h}$. The insoluble urea by-product was removed by filtration and the filtrate was concentrated under reduced pressure resulting in a foamy offwhite solid. Diisopropyl ethylamine (DIPEA, $27 \mu \mathrm{L}, 0.16$ $\mathrm{mmol}$ ) in dichloromethane (anhydrous, $1.5 \mathrm{~mL}$ ) and $126.9 \mathrm{mg}$, $0.1 \mathrm{mmol}$ ) were added to a portion of this resulting solid (82.9 $\mathrm{mg}, 0.1 \mathrm{mmol}$ ) and the mixture was stirred for $1 \mathrm{~h}$ under argon. Reaction completion was monitored by LC-MS (method 1, ESI $\dagger$ ). The solvent was removed under reduced pressure and the resulting residue was purified via CombiFlash (method 4 with A and B as solvents, ESI $\dagger$ ) to give 2 as a yellow semi-solid (91 mg, 95\% yield). ${ }^{1} \mathrm{H}$ NMR (500 $\left.\mathrm{MHz} \mathrm{CDCl}_{3}\right) \delta 7.43-7.27(\mathrm{~m}$, $5 \mathrm{H}), 6.45(\mathrm{~s}, 1 \mathrm{H}), 5.13(\mathrm{~s}, 2 \mathrm{H}), 3.76-3.55(\mathrm{~m}, 8 \mathrm{H}), 3.42-3.31(\mathrm{~m}$, $2 \mathrm{H}), 3.11(\mathrm{~m}, 8 \mathrm{H}), 3.00-2.82(\mathrm{~m}, 11 \mathrm{H}), 2.45(\mathrm{~m}, 2 \mathrm{H}), 2.12-1.98$ (m, 2H), 1.96-1.80 (m, 2H), 1.54-1.36 (m, 36H). ${ }^{13} \mathrm{C}$ NMR (126 $\left.\mathrm{MHz}, \mathrm{CDCl}_{3}\right) \delta 171.72,170.42,170.03,169.36,166.33,136.16$, 128.55, 128.48, 128.19, 128.15, 82.00, 81.73, 81.63, 66.85, 63.15, $56.30,55.60,55.28,52.97,52.36,50.58,44.69,41.10,29.41$, 28.28, 28.21, 28.13, 28.11, 24.46. LC-MS (method 1, ESI $\dagger$ ): $t_{\mathrm{R}}=$ $6.18 \mathrm{~min}$, HRMS: $m / z:[\mathrm{M}+\mathrm{H}]^{+}$calcd for $\left[\mathrm{C}_{47} \mathrm{H}_{80} \mathrm{~N}_{7} \mathrm{O}_{11}\right]^{+}$, 918.5916; found 918.5916 .

Synthesis of 3. Compound 2 ( $91 \mathrm{mg}, 0.1 \mathrm{mmol}$ ) was dissolved in dichloromethane $(1 \mathrm{~mL})$ along with trifluoroacetic acid (0.7 $\mathrm{mL}$ ) and stirred for $48 \mathrm{~h}$. Reaction progress was followed by LCMS. The solution was concentrated by rotary evaporation and the product was precipitated by addition of diethyl ether (10 $\mathrm{mL}$ ). The precipitate was isolated and washed repeatedly with diethyl ether then dried under vacuum. This material was used without further purification (56 mg, 81\% yield). ${ }^{1} \mathrm{H}$ NMR (500 $\left.\mathrm{MHz}, \mathrm{D}_{2} \mathrm{O}\right) \delta 7.42(\mathrm{~m}, 5 \mathrm{H}), 5.14$ (bs, 2H) 3.95-3.90 (m, 1H), 3.84, (m, 4H), 3.75-3.53 (m, 7H), 3.36 (m, 9H), 3.07 (m, 5H), 2.88 (m,
2H), 2.79 (m, 4H), $2.62(\mathrm{~m}, 1 \mathrm{H}), 1.94$ (bs, 2H). ${ }^{13} \mathrm{C}$ NMR (126 $\left.\mathrm{MHz}, \mathrm{D}_{2} \mathrm{O}\right) \delta 179.50,176.17,176.13$, 176.06, z160.10, 139.11, 131.79, 131.46, 130.73, 70.09, 59.31, 57.84, 57.46, 54.05, 53.66, 47.95, 47.82, 44.11, 32.89, 28.14. LC-MS (method 2, ESI $\dagger$ ): $t_{\mathrm{R}}=$ $3.55 \mathrm{~min}, \mathrm{HRMS}: \mathrm{m} / z$ : $[\mathrm{M}-\mathrm{H}]^{-}$calcd for $\left[\mathrm{C}_{31} \mathrm{H}_{46} \mathrm{~N}_{7} \mathrm{O}_{11}\right]^{+}$, 692.3255 found 692.3254 .

Synthesis of 4 . To a solution of $3(30 \mathrm{mg}, 0.04 \mathrm{mmol})$ dissolved in water $(1 \mathrm{~mL})$ was added gadolinium chloride $(20.9 \mathrm{mg}$, $0.06 \mathrm{mmol}$ ) and the $\mathrm{pH}$ was adjusted to 6.8 with $1 \mathrm{M} \mathrm{NaOH}$. The mixture was stirred overnight then purified by HPLC using method 5. The product was isolated as a white powder after freeze-drying (20.8 mg, 54\%). LC-MS (method 2, ESI $\dagger$ ): $t_{\mathrm{R}}=$ $8.01 \mathrm{~min}, \mathrm{~m} / \mathrm{z}$ : HRMS $[\mathrm{M}]^{+}$Calcd for $\left[\mathrm{C}_{31} \mathrm{H}_{44} \mathrm{GdN}_{7} \mathrm{O}_{11}\right]^{+}$, 848.2347; found 848.2356. Purity (>97\%) was assessed by LCICP-MS detecting gadolinium species as shown in Fig S1 (ESI $\dagger$ ).

Synthesis of Gd-CHyd (5). Compound 4 (40 mg) was dissolved in anhydrous methanol $(3 \mathrm{~mL})$ and activated palladium on carbon $(10 \%, 1 \mathrm{mg})$ was added to the solution. The suspension was placed under vacuum then connected to a balloon containing hydrogen gas. The reaction mixture was stirred for $18 \mathrm{~h}$ and progress was monitored by LC-MS. The reaction mixture was passed through a Celite pad and the filtrate was subjected to rotary evaporation. The resulting solid was purified by HPLC using method 5 . Purity and reactivity were confirmed using LC-ICP-MS in the presence or absence of butyraldehyde (method 6, $R_{\mathrm{t}}$ of $5: 14.1 \mathrm{~min} ; R_{\mathrm{t}}$ of butyraldehyde condensation product: $\mathbf{1 5 . 1} \mathrm{min}$. The gadolinium content was assessed by ICP-MS to determine the yield of the reaction (40.0 $\mu \mathrm{mol}, 87 \%$ isolated yield (30.1 $\mathrm{mg} 87 \%$.). LC-MS (method 3, $\mathrm{ESI} \dagger): \quad t_{\mathrm{R}}=8.01 \mathrm{~min}$, HRMS $\mathrm{m} / z$ : calcd for $[\mathrm{M}]^{+}$ $\left[\mathrm{C}_{23} \mathrm{H}_{37} \mathrm{GdN}_{7} \mathrm{O}_{9}\right]+$, 713.1899; found 713.1919. Purity (>98\%) was assessed by LC-ICP-MS with gadolinium detection as shown in Fig. S1 (ESI†).

\section{In vitro reaction rates}

First-order rate constants for reaction of Gd-Hyd and Gd-CHyd with 2-formylpyridine were determined by UV-vis spectroscopy as previously described..$^{24}$ The absorbance at $300 \mathrm{~nm}$ arising from the hydrazone condensation product was monitored in 30-60 second intervals for up to $3 \mathrm{~h}$. Reactions were conducted in phosphate-buffered saline ( $\mathrm{pH}$ 7.40) under pseudo-first order conditions with 2-formylpyridine at $1000 \mu \mathrm{M}$ and gadoliniumcontaining probes at $166 \mu \mathrm{M}$. Pseudo-first-order reaction kinetics were extracted from linear least-squares fits as previously described ${ }^{24}$ and reported as the mean of triplicate set of experiments \pm the standard deviation. Rates of reactions of allysine-targeted probes with 2-formyl pyridine were also determined using a HPLC with ICP-MS detection (ESI, Fig S5 and Table S1†).

\section{Determination of dissociation constants with LC-ICP-MS}

Dissociation constants were determined by incubating 2-formylpyridine (PBS, pH 7.40, $500 \mu \mathrm{M}$ ) with varying concentrations of Gd-Hyd or Gd-CHyd (10-300 $\mu \mathrm{M})$ for $72 \mathrm{~h}$ at $37^{\circ} \mathrm{C}$ and using LC-ICP-MS to determine ratios of Gd-containing species. The 
dissociation constant $\left(K_{\mathrm{d}}\right)$ is calculated as $K_{\mathrm{d}}=\frac{[A][B]}{[A B]}$ where $[A]$ is the concentration of unreacted 2-formylpyridine, $[B]$ is the concentration of unreacted probe (Gd-Hyd or Gd-CHyd) and $[A B]$ is the concentration of the resulting condensation product.

\section{Binding to porcine aorta and BSA-Ald}

Binding of Gd-CHyd and Gd-Hyd to protein and tissue aldehydes was carried out as previously described, ${ }^{18}$ with minor modifications as outlined in the ESI. $\dagger$ Solution of Gd-Hyd or Gd-CHyd ranging from 0.05 to $1 \mathrm{mM}$ were prepared in $\mathrm{PBS}(\mathrm{pH} 7.40)$ and $500 \mu \mathrm{L}$ aliquots were added to $25 \mathrm{mg}$ portions of purified aorta. The aorta segments were then incubated at $37^{\circ} \mathrm{C}$ for 72 hours. The aorta pieces were then washed twice with PBS (pH 7.40), placed in concentrated nitric acid overnight for digestion. The gadolinium content was then assessed by ICP-MS.

\section{Animal model}

All experiments and procedures were performed in accordance with the National Institutes of Health's "Guide for the Care and Use of Laboratory Animals" and were approved by the Massachusetts General Hospital Institutional Animal Care and Use Committee. C57Bl/6 adult male mice at 8 weeks of age (Jackson Laboratories, Barr Harbor, ME) received a single intratracheal dose of bleomycin, 1 units per $\mathrm{kg}$ body weight ( $50 \mu \mathrm{L}$ total volume) (Fresenius Kabi, Lake Zurich, Il) as previously described. ${ }^{36}$

MR imaging with Gd-Hyd was carried out 14 days after bleomycin injury $(N=8)$ and the same mice were imaged again 24 hours later with Gd-CHyd. An additional cohort of bleomycin-naïve mice $(N=4)$ were imaged with Gd-CHyd. All animals were dosed via tail vein injection at $100 \mathrm{nmol} \mathrm{g}^{-1}$ body weight from $30 \mathrm{mM}$ solutions of gadolinium probes as determined by ICP-MS. All animals were sacrificed after the imaging for biochemical analysis and histological evaluations

\section{MR images acquisition}

For all animals, images were acquired prior to, during, and post injection of probe. Animals were anesthetized with 1-2\% isoflurane and air/oxygen mixture to maintain constant respiration rate at 60 breaths/minute monitored by a small animal physiological monitoring system (SA Instruments Inc., Stony Brook $\mathrm{NY}$ ). Animals were placed in the scanner the following images were acquired: axial and coronal 2-dimensional (2D) Rapid Acquisition with Relaxation Enhancement (RARE) images, T1w 3D Fast Low Angle Shot (FLASH) images, and T1w 3D Ultra Short Time to Echo (UTE) images. During and immediately following $0.1 \mathrm{mmol} \mathrm{kg}^{-1}$ probe injection, a series of $10 \mathrm{~T} 1 \mathrm{w}$ FLASH images were acquired to monitor probe injection and clearance. UTE images were then acquired again at 15, 30 and 45 min post injection. Sequence parameter details are outlined in the ESI. $\dagger$

\section{MR lung image analysis}

We first visualized the vasculature of the lungs on the anatomical coronal images (T1-weighted RARE sequence) to identify lung parenchyma and exclude vessels and airways and to define the regions of interest (ROI). The FLASH image immediately post injection was also used to identify large vessels. ROIs of the lungs excluding large vessels and airways were drawn on eight sagittal slices. Additional ROIs were placed over the forearm muscle, the liver, and an ROI was placed in the field of view without any tissue to measure the variation in background (air) signal. These ROIs were then copied onto the UTE images to quantify signal intensity (SI). The analysis was repeated for the pre-probe and post-probe UTE data sets. Image visualization and quantification was performed using the Horos software package.

To quantify signal enhancement in the lung, contrast to noise ratio (CNR) was calculated as:

$$
\mathrm{CNR}=\left(\mathrm{SI}_{\text {lung }}-\mathrm{SI}_{\text {liver }}\right) / \mathrm{SD}_{\text {air }}
$$

Where $\mathrm{SI}=$ signal intensity, $\mathrm{SD}=$ standard deviation. An average of all image slices was calculated for the pre-injection images $\left(\mathrm{CNR}_{\text {pre }}\right)$ and for the post-injection images $\left(\mathrm{CNR}_{\text {post }}\right)$. The lung enhancement for each mouse is expressed as $\Delta \mathrm{CNR}$, the difference between the pre-injection CNR and the postinjection CNR

$$
\Delta \mathrm{CNR}=\mathrm{CNR}_{\text {post }}-\mathrm{CNR}_{\text {pre }}
$$

\section{MR lung image representation}

A difference image of pre and post contrast in the lungs was generated in Horos software by subtracting the UTE pre probe injection image from the UTE post probe injection image. A mask was applied to the lung based on the anatomical scan. The resulting difference image was then false colored, and superimposed on the corresponding anatomical (2D-RARE) image.

\section{Ex vivo tissue analyses}

Following imaging, animals were euthanized and the left lung was collected and homogenized, and then analyzed for allysine, hydroxyproline and gadolinium content. The right lung was harvested, fixed in neutral $10 \%$ buffered formalin, embedded in paraffin, and sectioned into $5 \mu \mathrm{m}$-thick slices for hematoxylin and eosin (H\&E) and sirius sed staining. Sirius red stained slides were scanned and images were digitally acquired and were analyzed by thresholding to determine the collagen proportional area (CPA), $\%$ area stained collagen positive, using ImageJ (National Institute of Health, Bethesda MD) as previously described..$^{18}$

\section{Conflicts of interest}

There are no conflicts to declare.

\section{Acknowledgements}

Research support from the National Heart Lung and Blood Institute (T32HL007208 to E. A. A.; R01HL116315, R01HL131907 to P. C.), the National Institute for Diabetes and Digestive, and Kidney Diseases (R01DK121789, U01DK104302 to P. C.) and the NIH Office of the Director (S10OD010650, S10OD025234 to P. C.) is gratefully acknowledged. 


\section{References}

1 M. L. Urban, L. Manenti and A. Vaglio, N. Engl. J. Med., 2015, 373, 95-96.

2 C. Kuhn, J. Boldt, T. E. King, E. Crouch, T. Vartio and J. A. McDonald, Am. Rev. Respir. Dis., 1989, 140, 1693-1703.

3 D. B. Esposito, S. Lanes, M. Donneyong, C. N. Holick, J. A. Lasky, D. Lederer, S. D. Nathan, S. O'Quinn, J. Parker and T. N. Tran, Am. J. Respir. Crit. Care Med., 2015, 192, 1200-1207.

4 G. Raghu, H. R. Collard, J. J. Egan, F. J. Martinez, J. Behr, K. K. Brown, T. V. Colby, J. F. Cordier, K. R. Flaherty, J. A. Lasky, D. A. Lynch, J. H. Ryu, J. J. Swigris, A. U. Wells, J. Ancochea, D. Bouros, C. Carvalho, U. Costabel, M. Ebina, D. M. Hansell, T. Johkoh, D. S. Kim, T. E. King, Y. Kondoh, J. Myers, N. L. Müller, A. G. Nicholson, L. Richeldi, M. Selman, R. F. Dudden, B. S. Griss, S. L. Protzko and H. J. Schünemann, Am. J. Respir. Crit. Care Med., 2011, 183, 788-824.

5 J. Hutchinson, A. Fogarty, R. Hubbard and T. McKeever, Eur. Respir. J., 2015, 46, 795-806.

6 D. A. Lynch, N. Sverzellati, W. D. Travis, K. K. Brown, T. V. Colby, J. R. Galvin, J. G. Goldin, D. M. Hansell, Y. Inoue, T. Johkoh, A. G. Nicholson, S. L. Knight, S. Raoof, L. Richeldi, C. J. Ryerson, J. H. Ryu and A. U. Wells, Lancet Respir. Med., 2018, 6, 138-153.

7 A. Levin, M. Tonelli, J. Bonventre, J. Coresh, J. A. Donner, A. B. Fogo, C. S. Fox, R. T. Gansevoort, H. J. L. Heerspink, M. Jardine, B. Kasiske, A. Köttgen, M. Kretzler, A. S. Levey, V. A. Luyckx, R. Mehta, O. Moe, G. Obrador, N. Pannu, C. R. Parikh, V. Perkovic, C. Pollock, P. Stenvinkel, K. R. Tuttle, D. C. Wheeler and K. U. Eckardt, Lancet, 2017, 390, 1888-1917.

8 B. Ley, H. R. Collard and T. E. King, Am. J. Respir. Crit. Care Med., 2011, 183, 431-440.

9 V. Cottin and L. Richeldi, Eur. Respir. Rev., 2014, 23, 106-110. 10 S. L. F. Walsh, Curr. Opin. Pulm. Med., 2018, 24, 445-452.

11 L. Richeldi, H. R. Collard and M. G. Jones, Lancet, 2017, 389, 1941-1952.

12 S. B. Montesi, P. Désogère, B. C. Fuchs and P. Caravan, J. Clin. Invest., 2019, 129, 24-33.

13 P. Désogère, S. B. Montesi and P. Caravan, Chem.-Eur. J., 2019, 25, 1128-1141.

14 R. Weissleder and U. Mahmood, Radiology, 2001, 219, 316333.

15 J. Wahsner, E. M. Gale, A. Rodríguez-Rodríguez and P. Caravan, Chem. Rev., 2019, 119, 957-1057.

16 J. M. Mäki, Histol. Histopathol., 2009, 24, 651-660.

17 M. L. Tanzer, Science, 1973, 180, 561-566.

18 H. H. Chen, P. A. Waghorn, L. Wei, L. F. Tapias, D. T. Schühle, N. J. Rotile, C. M. Jones, R. J. Looby, G. Zhao, J. M. Elliott, C. K. Probst, M. Mino-Kenudson, G. Y. Lauwers, A. M. Tager, K. K. Tanabe, M. Lanuti, B. C. Fuchs and P. Caravan, JCI Insight, 2017, 2, e91506.
19 P. A. Waghorn, C. M. Jones, N. J. Rotile, S. K. Koerner, D. S. Ferreira, H. H. Chen, C. K. Probst, A. M. Tager and P. Caravan, Angew. Chem., Int. Ed., 2017, 56, 9825-9828.

20 D. K. Kölmel and E. T. Kool, Chem. Rev., 2017, 117, 1035810376.

21 T. Dang, M. Suchy, Y. J. Truong, W. Oakden, W. W. Lam, C. Lazurko, G. Facey, G. J. Stanisz and A. J. Shuhendler, Chem.-Eur. J., 2018, 24, 9148-9156.

22 J. Wahsner, P. Désogère, E. Abston, K. A. Graham-O'Regan, J. Wang, N. J. Rotile, M. D. Schirmer, D. D. Santos Ferreira, J. Sui, B. C. Fuchs, M. Lanuti and P. Caravan, J. Am. Chem. Soc., 2019, 141, 5593-5596.

23 A. Kirby, M. Suchý, A. Brouwer and A. Shuhendler, Chem. Commun., 2019, 55, 5371-5374.

24 E. Kool, P. Crisalli and K. Chan, Org. Lett., 2014, 16, 14541457.

25 E. Kool, D. Park and P. Crisalli, J. Am. Chem. Soc., 2013, 135, 17663-17666.

26 A. Dirksen and P. E. Dawson, Bioconjugate Chem., 2008, 19, 2543-2548.

27 R. L. Levine, D. Garland, C. N. Oliver, A. Amici, I. Climent, A. G. Lenz, B. W. Ahn, S. Shaltiel and E. R. Stadtman, Methods Enzymol., 1990, 186, 464-478.

28 P. A. Waghorn, B. L. Oliveira, C. M. Jones, A. M. Tager and P. Caravan, J. Chromatogr. B: Anal. Technol. Biomed. Life Sci., 2017, 1064, 7-13.

29 S. R. Pinnell and G. R. Martin, Proc. Natl. Acad. Sci. U. S. A., 1968, 61, 708-716.

30 W. M. S. Russell, R. L. Burch and C. W. Hume, The principles of humane experimental technique, Altweb (Online service), Johns Hopkins Bloomberg School of Public Health., Johns Hopkins Center for Alternatives to Animal Testing and Universities Federation for Animal Welfare, 2005.

31 P. Caravan, Y. Yang, R. Zachariah, A. Schmitt, M. MinoKenudson, H. H. Chen, D. E. Sosnovik, G. Dai, B. C. Fuchs and M. Lanuti, Am. J. Respir. Cell Mol. Biol., 2013, 49, 1120-1126.

32 A. Rodríguez-Rodríguez, S. Shuvaev, N. Rotile, C. M. Jones, C. K. Probst, D. Dos Santos Ferreira, K. Graham-O Regan, E. Boros, R. S. Knipe, J. W. Griffith, A. M. Tager, A. Bogdanov and P. Caravan, ACS Sens., 2019, 4(9), 24122419.

33 S. B. Montesi, R. Rao, L. L. Liang, H. E. Goulart, A. Sharma, S. R. Digumarthy, B. S. Shea, R. T. Seethamraju, P. Caravan and A. M. Tager, Eur. Respir. J., 2018, 51, 1800171.

34 B. S. Shea, C. K. Probst, P. L. Brazee, N. J. Rotile, F. Blasi, P. H. Weinreb, K. E. Black, D. E. Sosnovik, E. M. Van Cott, S. M. Violette, P. Caravan and A. M. Tager, JCI Insight, 2017, 2, e86608.

35 S. Levy, V. Jacques, K. Zhou, S. Kalogeropoulos, K. Schumacher, J. Amedio, J. Scherer, S. Witowski, R. Lombardy and K. Koppetsch, Org. Process Res. Dev., 2009, 13, 535-542.

36 P. Desogere, L. Tapias, T. Rietz, N. Rotile, F. Blasi, H. Day, J. Elliott, B. Fuchs, M. Lanuti and P. Caravan, J. Nucl. Med., 2017, 58, 1991-1996. 